\title{
Ação dos térmitas no solo
}

\author{
Termites action on the soil
}

\section{Eric Victor de Oliveira Ferreira ${ }^{\mathrm{I}}$ Vanessa Martins ${ }^{\mathrm{II}}$ Alberto Vasconcellos Inda Junior ${ }^{\mathrm{III}}$ Elvio Giasson ${ }^{\text {III }}$ Paulo César do Nascimento ${ }^{\text {III }}$}

\section{- REVISÃO BIBLIOGRÁFICA -}

\section{RESUMO}

A ordem Isoptera é bastante conhecida pelo seu potencial como praga, apesar dos cupins-praga constituírem a minoria dentro do grupo. Os cupins (térmitas) são invertebrados dominantes em ambientes terrestres tropicais e estão espalhados desde as florestas úmidas até as savanas, sendo encontrados até mesmo em regiões áridas, nos mais variados habitats. Esses insetos têm um papel destacado e ainda pouco estudado nos ecossistemas tropicais. Ciclagem de nutrientes, aeração, infiltração de água do solo, bioturbação, formação de agregados e decomposição de material orgânico, são processos influenciados pela ação dos térmitas, que vão, direta ou indiretamente, influenciar a formação dos solos e da paisagem onde se encontram. Sugerimos que uma maior abordagem deva ser dirigida em futuras pesquisas para a influência desses insetos no solo sob condições específicas de uso e manejo, na produção sustentável de alimentos e nas mudanças climáticas.

Palavras-chave: Isoptera, atividade térmita, biologia do solo, bioturbação, cupins-praga.

\section{ABSTRACT}

The order Isoptera is well known by its potential as a plague, although the number of species that are plagues is small within the group. Termites are the dominant invertebrates in tropical terrestrial environments and are spread from tropical rainforests to the savannahs, being found even in arid regions, in various habitats. These insects have a major role and are still little studied in tropical ecosystems. Nutrient cycling, aeration, water infiltration of soil, bioturbation, aggregates formation and organic material decomposition, are processes influenced by the action of termites, which, directly or indirectly, affect soil and landscape formation wherever they are. We suggest that a better approach must be addressed in future researches about these insects influence in the soil under specified conditions of use and management, in sustainable food production and climate changes.

Key words: Isoptera, termite activity, soil biology, bioturbation, plague-termites.

\section{INTRODUÇÃO}

A importância ecológica dos isópteros em ecossistemas tropicais é grande, principalmente quando consideradas as modificações que podem causar no ambiente, desde alterações no aspecto visual da paisagem, pela construção de seus ninhos, além de alterações da topografia, como dos microrrelevos de murundus, até modificações nas propriedades físicas e químicas do solo, efeitos no processo de decomposição, ciclagem de nutrientes, entre outros (HOLT \& LEPAGE, 2000). Os cupins (térmitas) são a mais importante fauna do solo nos trópicos quentes sazonalmente secos (LOBRY DE BRUYN \& CONACHER, 1990), sendo fundamentais para o funcionamento do ecossistema, pois ocupam níveis tróficos na cadeia alimentar do solo (SILVA et al., 2007). Os térmitas são reconhecidos como "engenheiros do ecossistema" (DANGERFIELD et al., 1998), devido à habilidade que possuem de modificar a estrutura do

IPrograma de Pós-graduacão em Solos e Nutrição de Plantas, Universidade Federal de Viçosa (UFV), 36570-000, Viçosa, MG, Brasil. E-mail: ericsolos@yahoo.com.br. Autor para correspondência.

IIPrograma de Pós-graduacão em Solos e Nutrição de Plantas, Universidade Federal de Lavras (UFLA), Lavras, MG, Brasil.

IIIDepartamento de Solos, Faculdade de Agronomia, Universidade Federal do Rio Grande do Sul (UFRGS), Porto Alegre, RS, Brasil. 
hábitat, alterando a disponibilidade de recursos para outras espécies através de mudanças físicas em materiais bióticos ou abióticos.

Os cupins estão distribuídos na faixa de florestas tropicais e subtropicais, com uma maior riqueza de gêneros nas menores latitudes (EGGLETON et al., 1994). Eles são considerados organismos imprescindíveis à manutenção dos processos de decomposição e para os fluxos de carbono e nutrientes, devido principalmente à biomassa de suas populações e à variedade de seus hábitos alimentares (BANDEIRA $\&$ VASCONCELLOS, 2002).

Apesar dos benefícios físicos e químicos proporcionados por esses organismos no ecossistema, os cupins são mais conhecidos como pragas de pastagens e de madeira e por proporcionarem redução da área de plantio pela presença de seus ninhos (termiteiros) sobre a superfície do solo, dificultando também as práticas de manejo (GALLO et al., 2002), além da criação de microrrelevos do tipo murundus (RESENDE et al., 2007).

Devido à grande diversidade dos térmitas no Brasil e sua importância no ecossistema, principalmente nos tropicais, torna-se relevante salientar o papel deles, buscando informações a respeito da atuação desses insetos nos solos e no ambiente como um todo, contribuindo para a identificação de lacunas no conhecimento e, assim, nortear o avanço no desenvolvimento de futuras pesquisas.

Regiões de ocorrência e taxonomia

Os cupins são insetos sociais da ordem Isoptera, que contém cerca de 2.750 espécies conhecidas no mundo (CONSTANTINO, 2005), sendo estes invertebrados dominantes em ambientes terrestres tropicais e estão espalhados desde as florestas úmidas até as savanas, além de encontrados até mesmo em regiões áridas (EGGLETON et al., 1996). Atribui-se este fato à existência de simbiose com microorganismos, como bactérias, fungos e protozoários que auxiliam na sua alimentação, além de uma organização social bastante desenvolvida (COSTA-LEONARDO, 2002). Um exemplo da ocorrência dos térmitas no Brasil, além de citações em vários outros locais do país, é verificado na constatação de DIEHL et al. (2005) quando da presença de montículos de térmitas em áreas de zonas úmidas com pastagens anteriormente utilizadas com arrozais na costa Norte do Estado do Rio Grande do Sul.

Existem sete famílias representantes da ordem dos cupins: Mastotermitidae, Kalotermitidae, Termopsidae, Hodotermitidae, Serritermitidae,
Rhinotermitidae e Termitidae (GRASSE, 1986). Os cupins pertencentes às seis primeiras famílias são denominados "cupins inferiores", e os pertencentes à sétima (Termitidae) são denominados "cupins superiores". Tais designações não significam que esses insetos apresentam similaridades filogenéticas nem diferentes origens, referem-se apenas à dependência de protozoários flagelados simbiônticos, no caso dos "térmitas inferiores", para auxiliar na degradação da celulose (COSTA-LEONARDO, 2002). Termitidae é a família mais numerosa em espécies e a mais diversificada em termos ecológicos, compreendendo as subfamílias Macrotermitinae, Apicotermitinae, Termitinae e Nasutitermitinae (CANCELLO, 1989).

No Brasil ocorrem aproximadamente 300 espécies, que se distribuem entre as famílias Kalotermitidae, Rhinotermitidae, Termitidae e Serritermitidae. Os principais gêneros representantes da primeira família são Cryptotermes, Neotermes e Rugitermes, já na família Rhinotermitidae se destacam os gêneros Coptotermes e Heterotermes e, no caso da família Termitidae, os gêneros Cornitermes, Nasutitermes, Syntermes e Anoplotermes são os mais relevantes. Cornitermes é um gênero de cupins neotropicais presente em todo o país, sendo encontrado em formações florestais, cerrado, campos e pastagens (CONSTANTINO, 1999). A família Serritermitidae até recentemente continha uma única espécie, Serritermes serrifer, que ocorre apenas no Brasil, mas novas evidências indicam que Glossotermes oculatus, espécie da Amazônia, também pertence à Serritermitidae (ZANETTI et al., 2010).

Castas

$\mathrm{Na}$ sociedade dos cupins, existem indivíduos morfofisiologicamente diferentes, que compreendem as diferentes castas, responsáveis por diferentes tarefas. A casta estéril é representada pelos operários e soldados, sendo os primeiros os responsáveis pela busca e consumo imediato do alimento. Já os soldados atuam na defesa, enquanto a casta fértil, representada pelo casal real (rei e rainha), está envolvida nas atividades reprodutivas (KRISHNA, 1969). Os indivíduos alados (reprodutores) possuem dois pares de asas membranosas semelhantes, daí o nome da ordem (Iso $=$ igual; ptera $=$ asa). As asas possuem uma sutura basal característica, a qual favorece a queda das asas após o período reprodutivo, sobrando, adjacente ao corpo do inseto, uma escama, importante na taxonomia dos cupins. As operárias e os soldados dos cupins possuem uma depressão com poro frontal na cabeça, denominada fontanela, que é ligada a uma glândula cefálica e expele um líquido viscoso e 
espesso, com função de defesa. Outra característica importante de insetos dessa ordem é a associação mutualística existente com protozoários (Filo Mastigophora) que se localizam nos seus intestinos, responsáveis pela digestão do material celulósico ingerido pelo inseto. Anualmente (entre agosto e outubro), ocorre, nas colônias de cupins, um fenômeno conhecido como revoada, caracterizado pelo surgimento dos indivíduos reprodutores alados (siriris ou aleluias). Após a cópula, os machos e fêmeas, agora ápteros, tornam-se reis e rainhas e serão responsáveis pelo estabelecimento de novos ninhos (CARVALHO, 2004).

Ninhos

A construção de ninhos para abrigar a colônia representa uma das características dos insetos sociais (WILSON, 1971). Os ninhos de cupins, chamados de termiteiros, são classificados de acordo com sua posição em relação ao solo: ninhos arborícolas, construídos sobre ou dentro de árvores; ninhos hipógeos, construídos sob o solo; e ninhos epígeos, que possuem uma pequena parte subterrânea e a maior parte acima da superfície do solo. A maioria das espécies de Cornitermes constrói ninhos epígeos, sendo estes divididos em duas partes: uma camada externa resistente, construída principalmente de solo e uma câmara central celulósica (núcleo) onde vive a colônia. A camada externa auxilia na manutenção do microclima interno do ninho e funciona como defesa contra predadores (REDFORD, 1984). Os ninhos de Cornitermes cumulans, uma espécie construtora de ninhos epígeos comum no Cerrado, são utilizados como hábitat por vários animais, principalmente por outras espécies de cupins (SANTOS, 2008), demonstrando sua importância ecológica.

O microclima do termiteiro é relativamente estável e é mantido, em grande parte, pela ação dos cupins, pela arquitetura do ninho, pelo material utilizado para construção e principalmente pela interação e dinâmica destes fatores (REDFORD, 1984). Dentro dos ninhos, os térmitas são capazes de controlar a temperatura, umidade e atmosfera interna, e a localização e arquitetura destes ninhos determinam a regulação térmica; por exemplo, se eles estão expostos ao sol ou abaixo de dossel de floresta (NOIROT \& DARLINGTON, 2000). Para RATCLIFFE et al. (1952), a manutenção da umidade dentro do ninho é uma característica importante, considerando que os térmitas são extremamente sensíveis a trocas de umidade. A atmosfera interna baseia-se na estrutura de galerias e poros do ninho que permitem as trocas gasosas, sendo a sua ventilação essencial à manutenção da concentração adequada de oxigênio e carbono (NOIROT \& DARLINGTON, 2000).
Hábitos alimentares

A classificação dos térmitas em grupos tróficos foi feita de acordo com o trabalho de EGGLETON et al. (1995): cupins humívoros ou geófagos (que se alimentam de solo); cupins intermediários (que se alimentam da interface solo/ madeira); cupins xilófagos (que se alimentam de madeira); e cupins ceifadores (comedores de serapilheira) (REIS \& CANCELLO, 2007). No entanto, CONSTANTINO (1999) comenta que todas as espécies são consumidoras de serapilheira. Algumas das espécies de cupins que se alimentam de madeira tendem a ser polífagas e não consomem somente esse recurso, mas uma grande variedade de plantas vivas, fragmentos de plantas, esterco, além de muitos produtos armazenados e materiais usados pelo homem, sendo consideradas as maiores pragas em muitas áreas (HARRIS, 1970).

A dieta de madeira dos cupins demandou uma dependência coevolutiva de simbiontes do trato digestivo e de suas habilidades celulolíticas (CLEVELAND et al., 1934), que fizeram com que a sobreposição de gerações se tornasse uma necessidade" (THORNE, 1997). As plantas são, direta ou indiretamente, a fonte de recurso alimentar para os cupins, sendo importantes na abundância e na distribuição das espécies (LEE \& WOOD, 1971). Para os cupins humívoros, o alongamento de seu intestino sugere uma dieta de baixa qualidade (BIGNELL et al., 1983) e estão restritos aos hábitats com alta umidade, tais como florestas úmidas, e são sensíveis aos distúrbios ambientais (WOOD et al., 1982).

Ciclagem de nutrientes

Os cupins exercem um papel importante nos processos de formação de solo e ciclagem de nutrientes (EGGLETON et al., 1996; ANDERSON, 2005), sendo este último por meio da trituração, decomposição, humificação e mineralização de recursos celulósicos (COSTALEONARDO, 2002). Devidoà sua capacidade de digerir celulose, eles direcionam para si um considerável fluxo de energia, atingindo biomassa elevada e, ao mesmo tempo, servindo de alimento para um grande número de organismos (WOOD \& SANDS, 1978).

O acúmulo de material vegetal nos termiteiros, bem como o conteúdo fecal e a saliva utilizados na cimentação de suas paredes, tornam estes locais mais ricos em nutrientes e sais minerais (CASTRO JÚNIOR, 2002). A saliva dos cupins também tem a função de endurecer as paredes do cupinzeiro, dando a elas uma consistência quase pétrea (ZANETTI et al., 2010). Os térmitas são organismos que têm grande capacidade de concentrar nutrientes nos ninhos (SILVA 
\& MENDONÇA, 2007) e contribuem para a ciclagem de elementos, como, N, P, K, S, Ca, Na, C e O, com maior influência nos ciclos do Ce do N (LEE \& WOOD, 1971). Adicionalmente, esses insetos também interferem substancialmente nos processos atmosféricos por meio da liberação de metano $\left(\mathrm{CH}_{4}\right)$ e de dióxido de carbono $\left(\mathrm{CO}_{2}\right)$, devido ao processo de digestão da celulose, e através da fixação do $\mathrm{N}_{2}$ pela ação dos simbiontes intestinais (EGGLETON et al., 1999), influenciando diretamente nas condições climáticas. Como exemplo, estudos mostram que os térmitas podem ser responsáveis por até $2 \%$ do $\mathrm{CO}_{2}$ global e $15 \%$ do $\mathrm{CH}_{4}$ (BLACK \& OKWAKOL, 1997). Porém, salienta-se a necessidade do desenvolvimento de mais pesquisas nessa linha.

Avaliando a atividade de térmitas em pastagens, FUKUDA et al. (2003) verificaram taxas mais altas de ciclagem de $\mathrm{N}$ e maior frequência de pastejo nas áreas próximas aos termiteiros. Os cupins coletam partículas de diferentes profundidades do solo e as depositam em montículos, os quais são enriquecidos em C orgânico e nutrientes em relação aos solos adjacentes (BROSSARD et al., 2007). Tais incrementos se refletem na elevação do $\mathrm{pH}$ e da população microbiana nos montículos (OHKUMA, 2003). Posteriormente, o material acumulado é redistribuído por erosão, causando mudanças na estrutura e na fertilidade química do solo (SHAEFER, 2001; ANDERSON, 2005).

Em um estudo realizado no Estado de Santa Catarina, KASCHUK et al. (2006) observaram que os teores de $\mathrm{P}, \mathrm{K}, \mathrm{Ca}, \mathrm{Mg}$ e C orgânico foram maiores no centro dos ninhos em relação à área de solo adjacente. OHKUMA (2003) comenta que partículas de solo provavelmente sofrem modificações no trato intestinal devido ao $\mathrm{pH}$ extremamente alcalino, chegando a valores de até 12, fato que, conforme SHAEFER (2001), pode explicar o aumento da biodisponibilidade de $\mathrm{P}$ pela solubilização de $\mathrm{P}$ ligado a óxidos de $\mathrm{Fe}$ e Al.

\section{Bioturbação}

Desde o século XIX, o papel da biota do solo em revolvê-lo tem sido bem reconhecido, particularmente o dos térmitas (LEE \& WOOD, 1971). A ação de misturar e deslocar o material orgânico e mineral do solo, da superfície e do subsolo (bioturbação), pela fauna, é fundamental na dispersão de nutrientes ao longo do perfil do solo (PILLANS et al., 2002; SILVA \& MENDONÇA, 2007). Além das minhocas e minhocuçus, a bioturbação promovida pelas formigas e térmitas merece destaque na paisagem brasileira (RESENDE et al., 2007). Em regiões tropicais, a uniformidade dos perfis de solos, por exemplo, nos
Latossolos, é muitas vezes atribuída ao revolvimento do solo, com mistura de materiais de diferentes horizontes, o que tende a atenuar as características resultantes de processos pedogenéticos, como a lessivagem (DEMATTÊ et al., 1992). Nessa mesma linha, SCHAEFER (2001), trabalhando com amostras do horizonte B de Latossolos do Brasil, atribuiu a estrutura microgranular desses solos como sendo produto da ação da biota por longo tempo, e os térmitas do solo estão entre os principais organismos envolvidos em sua gênese. Este fato retrata a importância dos térmitas, ao relacioná-los com a gênese da principal classe de solo de nosso país, com destacado papel na produção de alimentos.

As taxas de acúmulo de solo resultante da atividade de térmitas variam de 0,05 a $0,4 \mathrm{~mm}^{2}$ ano ${ }^{-1}$ (LEE \&WOOD, 1971). HOLT \& LEPAGE (2000), por sua vez, estimaram que, para um período de $1000 \mathrm{anos}, 37 \mathrm{~cm}$ do subsolo poderiam ser translocados para a superfície pelos térmitas. KASCHUK et al. (2006) estimaram, para quatro municípios de Santa Catarina, uma amplitude de volume total de solo transportado pela atividade térmita entre 20,9 e $136,6 \mathrm{~m}^{3} \mathrm{ha}^{-1} \mathrm{e}$, desses, de $8,11 \mathrm{a}$ $63,3 \mathrm{~m}^{3} \mathrm{ha}^{-1}$ seriam transportados acima da superfície do solo.

Aeração e infiltração de água

O trabalho de ELDRIDGE (1994), para condições semiáridas australianas, enfoca o papel das estruturas de canais subterrâneos provenientes de termiteiros na capacidade de infiltração de água dos solos. Ele concluiu que a alta diversidade e biomassa de invertebrados em regiões semiáridas têm uma influência expressiva nas relações solo-água. Ao abrir túneis e construir seus ninhos em profundidades que podem atingir seis metros (LEPRUN, 1976), os cupins arejam e melhoram a estrutura do solo, além de movimentar verticalmente grande quantidade de partículas (CONSTANTINO, 2005). Alguns autores relacionam a confecção de galerias pelos térmitas com uma melhoria na porosidade e na capacidade de infiltração de água no solo (CANCELLO, 1989; MANDO, 1991; MIKLÓS, 1998; LEÓNARD \& RAJOT, 2001).

Estudos micromorfológicos indicam a participação de térmitas na gênese de microestrutura (escala microscópica) e modificação das características hidrológicas do solo (BARROS et al., 2001). Segundo GARNIER-SILLAM et al. (1991), a atividade dos térmitas pode provocar um incremento da porosidade (T. macrothorax, espécie humívora), principalmente nos horizontes superficiais, devido à ação mecânica da mastigação juntamente com o aumento da matéria orgânica. Porém, de acordo com estes mesmos autores, 
a ação dos térmitas pode causar, também, uma considerável redução na porosidade, com o desaparecimento completo de poros $>1,5 \mu \mathrm{m}$ por $\boldsymbol{M}$. Muller (espécie que cresce com cogumelos), todavia sem explicação deste fato pelos autores citados. Infiltração acumulada, taxas de infiltração, teor de água e porosidade do solo foram maiores em áreas com térmitas (MANDO et al., 1996). Isto sugere, de acordo com os autores, que os térmitas podem ser importantes agentes no controle de crosta de solo e na melhoria das suas propriedades físicas. Abaixo de chuva simulada foi demonstrado que as taxas de infiltração nos lugares com atividade térmita foram significativamente maiores $\left(88,4 \mathrm{~mm} \mathrm{~h}^{-1}\right)$ que em lugares sem essa atividade $\left(51,3 \mathrm{~mm} \mathrm{~h}^{-1}\right)$ (ELKINS et al., 1986). Apesar da apresentação destes dados, existem poucos estudos experimentais quantitativos sobre a influência dos térmitas na infiltração de água no solo (LÉONARD \& RAJOT, 2001), tornando-se necessário o desenvolvimento de mais pesquisas nessa área.

\section{Textura e mineralogia}

Os trabalhos que se referem à avaliação da textura dos solos com atividade térmita não são conclusivos, pois, em alguns casos, foram encontrados maiores teores de argila nos solos dos cupinzeiros em comparação aos solos adjacentes (BROSSARD et al., 2007) e, em outros, não se encontram diferenças (EGLER, 1984), demonstrando, assim, a necessidade de mais estudos nesse linha.

Estudos pioneiros apresentaram modificações na textura e em argilominerais do solo pela ação de térmitas (JOUQUET et al., 2002b). JOUQUET et al. (2002a) relatam que teores de argila em montículos de térmitas são normalmente $20 \%$ maiores que em solos próximos, mas não é sabido se térmitas selecionam partículas, se o solo é submetido a um fracionamento físico no tubo digestivo, ou se argilominerais 2:1 são modificados, gerando formas mais expansivas. É possível que minerais de argila sejam alterados quando partículas de solo são carreadas na boca dos térmitas ou em seus intestinos (KASCHUK et al., 2006).

Foi observado apenas menor teor de argila no centro do montículo, onde o teor de areia foi mais alto (KASCHUK et al., 2006). Provavelmente, segundo os autores, o maior teor de areia promove melhores condições para drenagem e controle da umidade e aeração no centro dos ninhos nos solos argilosos. Como micas formam vermiculita (ou esmectita) pelo intemperismo, liberando $\mathrm{K}^{+}$e substituindo outros cátions trocáveis, principalmente $\mathrm{Ca}^{+2}$ e $\mathrm{Mg}^{+2}$, foi especulado, por estes autores, que argilominerais 2:1, ricos em $\mathrm{K}^{+}$, seriam modificados durante a construção de seus montículos. Isso se daria pela extração de $\mathrm{K}^{+}$de argilominerais pelo metabolismo biológico de cupins que se alimentam de solo, já que cargas livres seriam neutralizadas com cátions da matéria orgânica, como o $\mathrm{Al}^{+3}$.

O teor de argila é mais alto em montículos de térmitas que em solo adjacente, provavelmente, devido às suas preferências por partículas mais finas de solos como um material cimentante para construção de seus ninhos (DONOVAN et al., 2001). Além da arquitetura, a seleção de partículas de solo na construção de ninhos já foi observada em cupins (gênero Odontotermis) cultivadores de fungos como forma de manutenção do microclima interno. Esses cupins constroem câmaras enriquecidas com partículas finas onde alojam os fungos. A capacidade de retenção de água do material usado na construção das câmaras é maior e, provavelmente, está relacionada à conservação de umidade e temperatura e ao crescimento dos fungos (JOUQUET et al., 2002a).

Por outro lado, ALMEIDA (2008) não verificou diferenças entre a porcentagem de argila na parede dos ninhos e aquela encontrada no solo das proximidades destes, o que também refuta a hipótese de seletividade de partículas finas. De acordo com a mesma autora, para cupins do gênero Cornitermes, é provável que estes não selecionem partículas do solo, mas, sim, o hábitat em que constroem seus ninhos. KASCHUK et al. (2006), em solos de Santa Catarina, também não encontraram diferenças no teor de argila entre o solo adjacente, o fundo e o topo dos montículos, além de constatarem que os térmitas não alteraram a composição mineralógica do solo estudado (argiloso) devido à alta estabilidade dos minerais de argila presentes neste solo, predominantemente compostos de caulinita e vermiculita com hidróxi-Al entre camadas (HIV).

\section{Térmitas como pragas}

A ordem Isoptera é bastante conhecida pelo seu potencial como praga, apesar dos cupins-praga constituírem a minoria dentro do grupo (cerca de 10\%) (LIMA et al., 2007). Com relação ao consumo de pastagens, CARVALHO (2004) enfatiza que há controvérsias entre os estudos, surgindo recentemente o conceito de que o cupim de montículo é uma "praga estética", pois apenas traria um aspecto visual desagradável ao produtor.

A ação prejudicial às pastagens pode ser atribuída a dois tipos de cupins, os construtores de ninhos epígeos, naquelas condições em que a densidade é elevada, e os consumidores de folhas vivas como alimento (FONTES, 1998). GALLO et al. (2002) relatam que os cupins de montículo constituem pragas importantes às pastagens, pois, além de estarem 
distribuídos em extensas áreas, seus ninhos dificultam os tratos culturais e agravam o processo de degradação das pastagens. No entanto, alguns trabalhos mostram que, mesmo em densidades elevadas desses cupinzeiros, a área reduzida é mínima. CZEPAK et al. (2003) observaram, em pastagens de 133 municípios no Estado de Goiás, um máximo de 500 cupinzeiros ha-1, os quais ocupavam apenas $3,9 \%$ da área útil. No entanto, existem relatos de densidade média variando de 6,2 até 1300 termiteiros ha-1 (BROSSARD et al., 2007), assim, apesar deste último caso ser de ocorrência esporádica, nessa magnitude pode ser prejudicial.

É questionável a redução de área útil das pastagens, como possível dano indireto atribuído aos cupins que formam montículos (VALÉRIO, 1995). Esse autor menciona que, em levantamentos realizados no Estado do Mato Grosso do Sul, a área média de cada cupinzeiro foi inferior a $0,5 \mathrm{~m}^{2}$. Acredita-se que o aspecto visual de uma pastagem parcialmente ocupada por cupinzeiros pode causar um efeito negativo: o produtor, muitas vezes, passa a combatê-los de maneira indiscriminada. Segundo FERNANDES et al. (1998), essas considerações são válidas, principalmente para Cornitermes spp. Algumas espécies do gênero Syntermes podem competir com o rebanho, especialmente no período seco, pois seus indivíduos danificam diretamente a pastagem, cortando e carregando grandes quantidades de folhas e colmos, tanto verdes como secos. Porém, COSENZA et al. (1974) concluíram que a eliminação do cupim de montículo não alterou a produção de matéria seca, nem a qualidade da pastagem, tampouco a cobertura vegetal.

Nos trópicos, os ninhos conspícuos (visíveis no hábitat) dos térmitas são elementos marcantes da composição estrutural dos ecossistemas (MARTIUS, 1994) e, de acordo com RESENDE et al. (2007), os termiteiros podem alterar substancialmente a topografia de certas áreas, originando, por exemplo, microrrelevos do tipo murundus. Estes microrrelevos apresentam, em geral, dimensões da ordem de 3 a $15 \mathrm{~m}$ de diâmetro à base, por uma altura que raramente excede a 3m (CORRÊA, 1989). Por fim, CONSTANTINO (2005) salienta a importância dos termiteiros, ao dizer que estes servem de abrigo a uma fauna diversa, incluindo artrópodes, vertebrados e outros grupos e que os ninhos velhos e abandonados servem de substrato para o desenvolvimento de várias espécies de plantas.

\section{CONCLUSÃO}

Devido ao relevante papel dos térmitas, tanto como agentes nos processos de formação do solo quanto como pragas (a minoria), principalmente nas regiões tropicais, salienta-se a necessidade de desenvolvimento de mais estudos sobre o tema. Percebe-se que há uma carência relativamente maior de informações a respeito dos processos que envolvem esses organismos em pesquisas relacionadas diretamente à Ciência do Solo, sendo estas, em sua maioria, atribuídas às áreas de zoologia e entomologia. Assim, sugerimos que uma maior abordagem deva ser dirigida, em futuras pesquisas, para a influência desses insetos no solo sob condições específicas de uso e manejo. Ademais, estudos que tratem de temas recorrentes, como produção sustentável de alimentos e mudanças climáticas, dentre outros, influenciados pela ação dos térmitas, terão demandas por parte da comunidade científica.

\section{REFERÊNCIAS}

ALMEIDA, J.S. Manutenção de temperatura e umidade e seletividade de partículas do solo em ninhos de Cornitermes (Isoptera: Termitidae) de uma floresta de terra firme da Amazônia Central. Disponível em: <http:/ /www.inpa.gov.br/ pdbff/cursos/efa/livro/2006/pdfs/ pfjuliana.pdf>. Online. Acesso em: 12 abr. 2008.

ANDERSON, M. Australian termites and nutrient recycling. Iowa: BIOL, 2005. 394p.

BANDEIRA, A.G.; VASCONCELLOS, A. A quantitative survey of termites in a gradient of disturbed highland forest in Northeastern Brazil (Isoptera). Sociobiology, v.39, p.429439, 2002.

BARROS, E. et al. The role of macrofauna in the transformation and reversibility of soil structure of an oxisol in the process of forest to pasture conversion. Geoderma, v.100, p.193-213, 2001 .

BIGNELL, D.E. et al. Structure, microbial associations and function of the so-called "mixed segment" of the gut in two soil-feeding termites, Procubitermes aburiensis and Cubitermes severus (Termitidae, Termitinae). Journal of Zoology, v.4, n.201, p.445-480, 1983 .

BLACK, H.I.J.; OKWAKOL, M.J.N. Agricultural intensification, soil biodiversity and agroecosystem function in the tropics: the role of termites. Applied Soil Ecology, v.6, p.37-53, 1997.

BROSSARD, M. et al. Nutrient storage in soils and nests of mound-building Trinervitermes termites in Central Burkina Faso: consequences for soil fertility. Biology and Fertility of Soils, v.43, p437-447, 2007.

CANCELlO, E.M. Revisão de Cornitermes Wasmann (Isoptera, Termitidae, Nasutitermitinae). 1989. $151 \mathrm{f}$. Tese (Doutorado em Zoologia) - Instituto de Biociências, Universidade de São Paulo, SP.

CARVALHO, G.A. Biologia e manejo integrado de cupins. Lavras: Departamento de Entomologia, Universidade Federal de Lavras, 2004. (Notas de aula da disciplina Entomologia aplicada (ENT-108). 
CASTRO JÚNIOR, P.R. Dinâmica da água em campos de Murundus do planalto dos Parecis. 2002. 193f. Tese (Doutorado em Geografia Física) - Programa de Pós-graduação em Geografia, Universidade de São Paulo, SP.

CZEPAK, C. et al. Ocorrência de espécies de cupins de montículo em pastagens no estado de Goiás. Pesquisa Agropecuária Tropical, v.33, n.1, p.35-38, 2003.

CLEVELAND, L. et al. The wood-feeding roach Cryptocercus, its Protozoa, and the symbiosis between Protozoa and roach. American Academy of Arts Sciences, v.17, p.185-342, 1934.

CONSTANTINO, R. Chave ilustrada para identificação dos gêneros de cupins (Insecta: Isoptera) que ocorrem no Brasil. Papéis avulsos de Zoologia, v.40, p.387-448, 1999.

CONSTANTINO, R. Padrões de diversidade e endemismo de térmitas no bioma Cerrado. In: SCARIOT, A.O. et al. (Eds.). Biodiversidade, ecologia, e conservação do cerrado. Brasília: Ministério do Meio Ambiente, 2005. p.319- 333.

CORRÊA, G.F. Les microreliefs "murundus" et leur environnement pédologique dans I' ouest du Minas Gerais, région du Plateau Central Brésilien. 1989. 144f. Tese (Doutorado em Solos) - Université de Nancy I, Vandoeuvreles-Nancy.

COSEnZA, G.W.; CARVAlHo, M.M. Controle e nível de dano do cupim de montículo em pastagens. Revista Brasileira de Zootecnia, v.3, n.1, p.1-12, 1974.

COSTA-LEONARDO, A.M. Cupins-praga: morfologia, biologia e controle. Rio Claro-SP: Divisa, 2002. 128p.

DEMATTÊ, J.L.I. et al. Influência da drenagem na morfogênese de solos desenvolvidos de rochas básicas no município de Piracicaba (SP). Revista Brasileira de Ciência do Solo, v.16, p.241-248, 1992.

DIEHL, E. et al. Formigas e térmitas co-habitantes dos murundus em zonas úmidas de Santo Antonio da Patrulha, Rio Grande do Sul, Brasil. Brazilian Journal of Biology, v.65, n.3, p.431437, 2005. Disponível em: <http://www.scielo.br/cgi-bin/ wxis.exe/iah/>. Acesso em: 10 dez. 2009. doi: 10.1590/S151969842005000300008 .

DANGERFIELD, J.M. et al. The mound-building termite Macrotermes michaelseni as an ecosystem engineer. Journal of Tropical Ecology, v.14, p.507-520, 1998.

DONOVAN, S.E. et al. The effect of a soil feeding termite, Cubitermes fungifaber (Isoptera: Termitidae) on soil properties: termites may be an important source of soil microhabitat heterogeneity in tropical forests. Pedobiologia, v.45, p.111, 2001. Disponível em: <http://www.sciencedirect.com/ science?_ob=ArticleURL\&_udi=B 7CW5-4DS314W$1 \mathrm{P} \&$ _user $=10 \&$ \&coverDate $=12 \% 2 \mathrm{~F} 31 \% 2 \mathrm{~F} 2001 \&$ \&doc $=1 \&$ fm $\mathrm{t}=$ high \&_orig $=$ search $\&$ _origin $=$ search $\&$ _sort $=\mathrm{d} \&$ _docanchor $=$ \&view=c\&_acct $=C 000050221 \& \_v e r s i o n=1 \& \_u r l$ Version $=0$ \&_userid $=$ $10 \& \mathrm{md} 5=3441950 \mathrm{~d} 6 \mathrm{e} 954677 \mathrm{dab} 76 \mathrm{cfa} 7 \mathrm{~b} 8 \mathrm{edf} 8 \mathrm{f} \&$ searchtype $=\mathrm{a}>$. Acesso em: 05 fev. 2010. doi:10.1078/0031-4056-00063.

EGGLETON, P. et al. The species richness (Isoptera) under differing levels of forest disturbance in the Mbalmayo Forest
Reserve, southern Camerron. Journal of Tropical Ecology, v.11, p.85-98, 1995.

EGGLETON, P. et al. Explaining global diversity: productivity or history. Biodiversity and Conservation, n.27, p.318330,1994

EGGLETON, P. et al. Termite assemblage, forest disturbance and greenhouse gas fluxes in Sabah, East Malaysia. Philosophical Transactions of the Royal Society of London, v.354, p.1791-1802, 1999.

EGGLETON, P. et al. The diversity, abundance and biomass of termites under differing levels of disturbance in the Mbalmayo Forest Reserve, southern Cameroon. Philosophical Transactions of the Royal Society of London, v.351, p.5168. 1996.

EGLER, I. Importância dos cupinzeiros de Procornitermes araujoi (Isoptera, Termitidae) na ciclagem de nutrientes em um cerrado de Brasília. 1984. 98f. Dissertação (Mestrado em Ecologia) - Programa de Pós-graduação em Ecologia, Universidade de Brasília, DF.

ELDRIDGE, D. Nests of ants and termites influence infiltration in a semiarid woodland. Pedobiologia, v.38, p.481-492, 1994.

ELKINS, N.Z. et al. The influence of subterranean termites on the hydrological characteristics of a Chihuahuan desert ecosystem. Oecologia, v.68, p.521-528, 1986.

FERNANDES, P.M. et al. Cupins de montículos em pastagens: prejuízo real ou praga estética? In: FONTES, L.R.; BERTI FILHO, E. (Eds.). Cupins: o desafio do conhecimento. Piracicaba-SP: FEALQ, 1998. p.187-210.

FONTES, L.R. Cupins nas pastagens do Brasil: algumas indicações de controle. In: FONTES, L.R.; BERTI FILHO, E. (Eds.). Cupins: o desafio do conhecimento. PiracicabaSP: FEALQ, 1998. p.211-225.

FUKUDA, E. et al. Effects of mounds of a termite Cornitermes cumulans on grass production and behavior of grazing cattle in semitropical grasslands in Brazil. Grassland Science, v.49, p.24-25, 2003

GALLO, D. et al. Entomologia agrícola. Piracicaba-SP: FEALQ, 2002. 920p.

GARNIER-SILLAN, E. et al. Rôle des termites sure le spectre poral des sols forstiers tropicaux. Insects Sociaux, v.38, p.397$412,1991$.

GRASSÉ, P.P. Termitologia. Paris: Masson, 1986. Tomo 3.

HARRIS, W.V. Termites of the Palaeartic region. In: KRISHNA, K.; WEESNER, F.M. (Eds.). Biology of termites. New York: Academic, 1970. Cap.2, p.295-313.

HOLT, J.A.; LEPAGE, M. Termites and soil properties. In: ABE, T. et al. (Eds.). Termites, evolution, sociality, symbiosis, ecology. Dordrecht: Kluwer Academic, 2000. p.389-407.

JOUQUET, P. et al. Termite soil preferences and particle selections: strategies related to ecological requirements. Insectes Sociaux, v.49, p.1-7, 2002a. 
JOUQUET, P. et al. Effect of termites on clay minerals in tropical soils: fungus-growing termites as weathering agents. European Journal of Soil Science, v.53, p.521-527, 2002b.

KASCHUK, G. et al. Termite activity in relation to natural grassland soil attributes. Scientia Agricola, v.63, n. 6, p.583588 , 2006. Disponível em: <http://www.scielo.br/ scielo.php?script =sci_abstract \& pid=S 0103 $90162006000600013 \& \operatorname{lng}=e n \& n r m=i s o \& t \operatorname{lng}=p t>$. Acesso em: 12 jun. 2008. doi: 10.1590/S0103-90162006000600013.

KRISHNA, K. Introduction. In: KRISHNA, K.; WEESNER, F.M. (Eds.). Biology of termites. New York: Academic, 1969. V.1, p.1-17.

LEE, K.E.; WOOD, T.G. Termites and soils. London and New York: Academic, 1971. 251p.

LEE, K.E.; WOOD, T.G. Physical and chemical effects on soils of some Australian termites, and their pedological significance. Pedobiologia, v.11, p.376-409, 1971.

LÉONARD, J.; RAJOT, J.L. Influence of termites on runoff and infiltration: quantification and analysis. Geoderma, v.104, p.17-40, 2001

LEPRUN, J.C. Une construction originale hypogée pour le stockage de l'eau par les Termites en régions sahelo-soudaniennes de Haute-Volta. Pedobiologia, v.16, p.451-456, 1976.

LIMA, J.T.; COSTA-LEONARDO, A.M. Recursos alimentares explorados pelos cupins (Insecta: Isoptera). Biota Neotropica, v.7, n.2, p.243-250. 2007.

LOBRY DE BRUYN, L.A.; CONACHER, A.J. The role of termites and ants in soil modification: a review. Australian Journal of Soil Research, v.28, p.55-93, 1990.

MANDO, A. Impact de l' activite des termites sur la degradation de la biomasse vegetale et quelques proprietes physiques des sols degrades: Etude mente a Zanamogo (Burkina Faso). Zogona: Université de Ouagadougou, ISN/IDR, 1991. 76p. (Mémoire de fin d'études).

MANDO, A. et al. Effects of termites on infiltration into crusted soil. Geoderma, v.74, p.107-113, 1996.

MARTIUS, C. Diversity and ecology of termites in Amazonian forest. Pedobiology, v.38, p.407-428, 1994.

MIKLÓS, A.A.W. Papel de cupins e formigas na organização e na dinâmica da cobertura pedológica. In: FONTES, L.R.; BERTI FILHO, E. (Eds.). Cupins: o desafio do conhecimento. Piracicaba: FEALQ, 1998. p.227-241.

NOIROT, C; DARLINGTON, J.P.E.C. Termite nests: architecture, regulation and defence. In: ABE, T. et al. (Eds.). Termites: evolution, sociality, symbioses, ecology. Netherlands: Kluwer Academic, 2000. p.121-139.

OHKUMA, M. Termite symbiotic systems: efficient biorecycling of lignocellulose. Applied and Environmental Microbiology, v.61, p.1-9, 2003.

PILLANS, B. et al. The dynamics of soils in north Queensland: rates of mixing by termites determined by single grain luminescence dating. In: ROACH, I.C. (Ed.). Regolith and Landscapes in Eastern Australia. Canberra: CRC LEME, 2002. p.100-101.

RATCLIFFE, F.N. et al. Australian termites: the biology, recognition and economic importance of the common species. Melbourne, Australia: CSIRO, 1952. 124p.

REDFORD, K.H. The termitaria of Cornitermes cumulans (Isoptera, Termitidae) and their role in determining a potential keystone species. Biotropica, v.16, p.112-119, 1984.

REIS, Y.T.; CANCELLO, E.M. Riqueza de cupins (Insecta, Isoptera) em áreas de Mata Atlântica primária e secundária do sudeste da Bahia. Série Zoologia, v.97, p.229-234, 2007.

RESENDE, M. et al. Pedologia: base para distinção dos ambientes. 5.ed. rev. Lavras- MG: UFLA, 2007. 322p.

SANTOS, T. Associação entre Cornitermes spp. (Isoptera: Termitidae) e cupins inquilinos em uma área de floresta de terra firme na Amazônia Central. Disponível em: <http:/ /www.inpa.gov.br/ pdbff/download/efa/livro/2006/pdfs/ pfthiago.pdf>. Online. Acesso em: 15 jun. 2008.

SHAEFER, C.E. Brazilian latosols and their B horizon microstructure as long-term biotic constructs. Australian Journal of Soil Research, v.39, p.909-926, 2001.

SILVA, I.R; MENDONÇA, E.S. Matéria orgânica do solo. In: NOVAIS, R.F. et al. (Eds.). Fertilidade do solo. Viçosa: SBCS, 2007. p.275-374.

SILVA, R.F. et al. Macrofauna invertebrada edáfica em cultivo de mandioca sob sistemas de cobertura do solo. Pesquisa Agropecuária Brasileira, v.42, p.865-871, 2007. Disponível em: 〈http://www.scielo.br/pdf/pab/v42n6/v42n6a14.pdf〉. Acesso em: 23 set. 2009. doi: 10.1590/S0100-204X2007000600014.

THORNE, B.L. Evolution of eusociality in termites. Annual Review of Ecology and Systematics, v.28, p.27-54, 1997.

VALÉRIO, J.R. Ocorrência, danos e controle de cupins de montículo em pastagens. In: REUNIÃO SUL-BRASILEIRA DE INSETOS DE SOLO, 1995, Dourados, MS. Ata e Resumos... Dourados: EMBRAPA-CPAO, 1995. V.5. 110p. p.33-36.

WILSON, E.O. The insect societies. Cambridge: Belknap, 1971. 548p.

WOOD, T.G.; SANDS, W.A. The role of termites in ecosystems. In: BRIAN, M.V. (Eds.). Production ecology of ants and termites. Cambridge: Cambridge University, 1978. p.245292.

WOOD, T.G. et al. Abundance and distribution of termites (Isoptera) in a riparian forest in the southern Guinea savanna vegetation zone of Nigeria. Biotropica, v.14, p.25-39, 1982.

ZANETTI, R. et al. Manejo integrado de cupins. Lavras: Departamento de Entomologia, Universidade Federal de Lavras, 2010. (Notas de aula da disciplina Entomologia aplicada (ENT-109). 